\title{
Vitamin B12 Gene Polymorphisms and Chronic Diseases
}

\author{
Anjali Haloi ${ }^{1}$ and Debabrata Das ${ }^{2^{*}}$ \\ ${ }^{1}$ Senior Technical Assistant, North-Eastern Hill University, Shillong, India \\ ${ }^{2}$ Research Fellow, Dr. B. Borooah Cancer Institute, Guwahati, India
}

*Corresponding author: Debabrata Das, Research Fellow, Dr. B. Borooah Cancer Institute, Guwahati - 781016, India; Phone: +919954175250; Fax: +91-361-2472636; E-mail: drdasdebabrata@gmail.com

Received date: Jul 28, 2014, Accepted date: Sep 28, 2014, published date: Oct 6, 2014

Copyright: ( 2014 Das D .et al. This is an open-access article distributed under the terms of the Creative Commons Attribution License, which permits unrestricted use, distribution, and reproduction in any medium, provided the original author and source are credited.

\begin{abstract}
Vitamin B12 or cobalamin is an essential nutrient with important roles in DNA synthesis, repair and methylation. It is also required in the one carbon metabolism pathway to reduce plasma homocysteine concentrations. Several epidemiological studies have indicated that genes and metabolites of the B vitamin-mediated one-carbon metabolic pathway are associated with chronic diseases. This short review describes polymorphisms in the MTHFR, FUT2 and TCN2 genes which have been implicated in cardiovascular diseases and neural tube defects amongst others.
\end{abstract}

\section{Introduction}

Vitamin B12, a water-soluble vitamin, belongs to a group of complex molecules that have cobalt containing corrin ring and are hence also called cobalamins. Vitamin B12, as also other B vitamins, play a key role as enzyme cofactors or substrates in one-carbon metabolism whereby folate transfers one-carbon groups in a range of biological processes including DNA synthesis, methylation, and homocysteine metabolism [1]. For instance, Vitamin B12 as a cofactor for methionine synthase catalyzes the conversion of homocysteine to methionine which is an intermediate in the formation of $\mathrm{S}$ adenosylmethionine. The latter is a universal methyl donor for about 100 different substrates, including DNA, RNA, hormones, proteins and lipids. It is also a cofactor for L-methylmalonyl-CoA mutase which converts L-methlmalonyl-CoA to succinly-CoA in the degradation of propionate, as essential biochemical reaction in metabolism of fat and protein [2].

The nutritional deficiency of vitamin B12 results in pernicious anaemia and neurological disorders. Subclinical deficiencies during pregnancy have been linked with neural tube defects and in adults it has been associated with increased risk of coronary artery disease and some cancers such as colorectal cancer. The prime hypothesis for the protective effect of $\mathrm{B}$ vitamins is that deficiencies increase the production and decrease the catabolism of homocysteine, which is an established risk factor for coronary artery diseases. Homocysteine affects various proatherogenic processes including inflammation, thrombosis, endothelial dysfunction, and vascular smooth muscle cell proliferation [1].

Deficiencies of folate and vitamin B12 have been found to be associated with high levels of plasma homocysteine. Circulating levels of $\mathrm{B}$ vitamins and homocysteine are at least in part genetically determined [1]. The study of gene polymorphisms and their effect on the ability to digest, absorb, and utilize vitamin B12 is imperative for a proper understanding of the interaction of diet, genetics, and health. Recent genome wide association studies have identified potential loci that influence plasma vitamin B12 levels. This article describes some of the common gene variants which are implicated in vitamin B12 associated chronic diseases.

\section{MTHFR}

A thermolabile variant with decreased specific activity of the enzyme 5,10-Methylenetetrahydrofolate Reductase (MTHFR) was first described by Kang et al (1988). In later studies (Kang et al 1991) they were able to establish that thermolability of MTHFR is inherited as an autosomal recessive trait. Genetic analyses by Frosst et al (1995) demonstrated that a 677C-T transition in the MTHFR coding sequence, which changed a highly conserved alanine into a valine residue $(\mathrm{A} 222 \mathrm{~V})$, is responsible for the thermolabile phenotype [see 3].

This can lead to lower levels of serum folate, increased levels of plasma homocysteine, and genomic DNA hypomethylation, particularly in those with a diet low in folate. Biochemically, the A222V variant may be less tightly bound to its flavin cofactor and more prone to dissociation into monomers but can be stabilized by reduced folates [3].

Because this variant can be linked to serum homocysteine, clinical biomarkers, the $677 \mathrm{C}-\mathrm{T}$ mutation has been investigated in many epidemiological disease association studies. Various biologic data and the epidemiologic evidence suggest that homocysteine itself is the cause, rather than an epiphenomenon, of arteriosclerosis [4]. Kang et al [5] first reported a more-than-threefold increase in the homozygous frequency of the MTHFR polymorphism among patients with coronary artery disease, compared with controls ( $17 \%$ vs. $5 \%)$, and demonstrated that the elevated homocysteine levels associated with the MTHFR variant could be reduced by folic-acid administration. Similar association was demonstrated by Kluijtmans et al [6] who reported the homozygous mutation in the MTHFR gene is associated with a threefold increase in risk for premature cardiovascular disease in Caucasian subjects. Homozygosity for the $677 \mathrm{C}-\mathrm{T}$ mutation in the MTHFR gene was found in 9 (15\%) of 60 cardiovascular patients and in only $6(\sim 5 \%)$ of 111 control individuals (odds ratio 3.1). While various studies in the West have shown that 677T MTHFR allele is associated with increased levels of plasma homocysteine in ethnic Europeans [7], it has been suggested that genetic determinants of plasma homocysteine in Asians vary to a great extent. T-allele frequency for C677T mutation of MTHFR gene in the South Indian population (0.10) is lower compared to UK (0.186) and USA (0.322) and much higher when compared to Sri Lanka (0.049) [8]. A recent 
Page 2 of 5

study carried out in Singapore indicates that nationals of Indian descent had the highest frequency of CBS 844ins68 allele and MS 2756G allele, while Singaporean Chinese had the highest frequency of MTHFR 677T allele [9]. The average changes in homocysteine levels of a Pakastani population for MTHFR 677CT and TT genotypes were positive $[\beta$ (SE $\beta$ ), 2.01(0.63) and $16.19(1.8) \mathrm{mmol} / \mathrm{L}$, respectively].It was reported that individuals with either CT or TT variant of MTHFR 677 along with folate deficiency have nearly 5 times increased risk of hyperhomocysteinemia, while risk of hyperhomocysteinemia increases 4.33 fold in individuals carrying CT or TT variant of MTHFR 677 with vitamin B 12 deficiency. Gene polymorphism (especially MTHFR C677T transition), folate and vitamin B12 deficiencies, male gender and high blood lead level appear to be contributing towards the development of hyperhomocysteinemia in a Pakistani population [10].

Past studies have investigated the association between the C677T MTHFR polymorphism and Neural Tube Defect (NTD) susceptibility. A meta-analysis of all studies published until January 2012 (2429 cases and 3570 controls) to investigate the association between the maternal MTHFR C677T polymorphism and risk of NTDs found that mothers with the homozygous TT genotype showed a significantly increased NTD risk compared with homozygous CC genotype carriers (with pooled odds ratio 2.022) [11].

The other frequent MTHFR mutation in exon 7, the A1298C transversion, is not associated with thermolability of the enzyme with no impact on plasma total homocysteine. This mutation causes a glutamate to alanine substitution in the MTHFR protein, abolishes an MboII recognition site, and has an allele frequency of 0.33 . This $\mathrm{A}$ to $\mathrm{C}$ mutation at position 1298 , results like the $677(\mathrm{C}$ to $\mathrm{T}$ ) mutation in a decreased MTHFR activity (ANOVA $\mathrm{P}<.0001$ ), which is more pronounced in the homozygous than the heterozygous state. The 677(C to T) lies in the catalytic domain of the protein, whereas the 1298(A to C) is located in the presumed regulatory domain [12].

In a study of Dutch patients with NTD and their parents, it was reported that heterozygosity for both mutations (677 C to T and 1298 A to C) resulted in an even lower MTHFR activity than heterozygosity for either of the MTHFR mutations separately; resulting in significantly elevated Hcy and decreased plasma folate levels. The frequency of the 1298(A to C) mutation in NTD-affected children tended to be increased, which also suggests that this mutation is a genetic risk factor for this developmental anomaly. Especially under conditions of low intake of folates or during high requirements of folate, like pregnancy, this mutation could become of clinical importance [12].

Many subsequent epidemiologic studies have evaluated the association between MTHFR A1298C polymorphism and NTD risk, but with inconsistent results. A positive association between A1298C and NTD has not been conclusively established. A recent metanalysis by Wang et al [13] that included literature published in PubMed, Embase, and Medline database concluded that A1298C polymorphism is not associated with neural tube defect susceptibility in the white population. They however concluded that further investigations in this area are required to get more insight into the role of MTHFR A1298C polymorphism in NTD, particularly in specific type of NTD risk. It is also necessary to address the effects of combined heterozygote (CT/AC) on NTD cases. Taking the severe prevalence and high mortality of NTD into account, this is an area which is of tremendous significance.

\section{FUT2}

The human $\alpha(1,2)$ - and $\alpha(1,3)$-fucosyltransferase genes constitute a multigene family, and seven genes encoding human fucosyltransferases (FUT1-FUT7). The a $(1,2)$ fucosyltransferase plays a key role for tissue expression of the $\mathrm{H}$ antigen-a common precursor for the blood group A and B antigens. The FUT1 gene isolated by Larsen [14] encodes $H$ type a $(1,2)$ fucosyltransferase ( $H$ enzyme) that regulates the expression of the $\mathrm{H}$ antigen and thereafter $\mathrm{A}$ and/or $\mathrm{B}$ antigens in the erythroid lineage and in the vascular endothelia. FUT2 corresponding to the human Secretor locus [15] encodes Secretor type $\alpha(1,2)$ fucosyltransferase (Se enzyme) that regulates the expression of these antigens in the secretory glands and in the digestive mucosa. About $20 \%$ of individuals are non-secretors who fail to express the $\mathrm{ABO}$ antigen in saliva, because they are homozygous for the null allele of FUT2.

The FUT2 gene is highly polymorphic and show differential ethnic prevalence [16]. To identify loci associated with plasma vitamin B12, homocysteine, folate and vitamin B6 (active form pyridoxal 50phosphate, PLP), Hazra et al [17] conducted a meta-analysis of three genome wide association scans $(\mathrm{n}=4763)$. On chromosome $19 \mathrm{q} 13$, they confirmed the association of plasma vitamin B12 with rs602662 and rs492602 ( $\mathrm{P}$ value $=1.83 \times 10-15$ and $1.30 \times 10-14$, respectively) in strong linkage disequilibrium (LD) with $\operatorname{rs601338}(\mathrm{P}=6.92 \mathrm{x}$ 10-15), the FUT2 W143X nonsense mutation and determinant of FUT2 secretor status. Participants homozygous for non-secretor variants had higher B12 levels than carriers of the secretor genotype. The authors had, in an earlier study [18] shown that white women who were homozygous FUT2 rs492602 G, which is in strong linkage disequilibrium with the FUT2 rs601338, also known as $428 \mathrm{G} / \mathrm{A}$ nonsecretor variant allele (W143X variant), had higher plasma vitamin B-12 concentrations.

The rs6022662 locus was the top SNP $(\mathrm{p}=2.83 \mathrm{x}$ 10-20) implicated in a meta-analysis of genome wide association study from Italy (Tuscany and Sardinia) and USA (Baltimore-Washington DC). The presence of the A allele was associated with $44.2 \mathrm{pg} / \mathrm{mL}$ higher vitamin B12 concentrations [1].

The common genetic determinants of vitamin B12 levels in nonEuropean populations are not well known. Lin et al [19] performed a two-stage genome wide association studies (1999 and 1496 healthy Chinese men in the first and second stage, respectively) to identify genetic loci that were associated with serum level of Vitamin B12 levels in the Chinese population. They identified four novel genomic loci that were significantly associated with serum level of vitamin B12 including MS4A3, CLYBL, FUT6 and 5q32 region. For the FUT2 gene, three SNPs, including rs602662, rs492602 and rs601338, previously reported to be associated with vitamin B12 levels were not confirmed in the first stage. In contrast, another non-synonymous SNP in the FUT2 gene (rs10447781) was strongly associated with vitamin B12 levels, with a combined P-value of $1.63 \times 10-35$. The SNP was in weak linkage disequilibrium $(\mathrm{r} 2=0.001)$ with any of the above three reported SNPs in the Chinese population. In addition, rs1047781 was not polymorphic in Europeans. The substitution of A to T allele leads to an amino acid change from isoleucine to phenylalanine in Asian populations. The authors suggest that rs1047781 is plausibly the potential functional variant that is associated with vitamin B12 level in the Asian population.

The human secretor (Se) blood group is determined by polymorphisms in FUT2 through the expression of a1,2- 
fucosyltransferase that mediates the fucosylation of oligosaccharides to form $\mathrm{H}$ type 1 and 2 antigens. Gastric pathogens such as Helicobactor pylori adhere to the gastric and duodenal mucosa with the help of these $\mathrm{H}$ antigens. Overgrowth of gastric bacteria, such as with $\mathrm{H}$. pylori, has been associated with vitamin B12 deficiency. Interestingly, rs602662 has been identified in non-secretor status, or the absence of $\mathrm{H}$ antigens, in individuals from Northern Portugal. The reduced activity of the FUT2 enzyme with the A allele may decrease susceptibility to bacterial infection and indirectly lower the risk of vitamin B12 mal-absorption, thereby resulting in higher vitamin B12 concentrations in A allele carriers [1].

It has been suggested that during the gastric phase of vitamin B12 absorption, the low $\mathrm{pH}$ of the stomach and the peptic acid digestion of food protein-bound cobalamin are necessary for the release of cobalamin. H. pylori-induced chronic atrophic gastritis may cause a disturbance of acid/peptic secretion, which can in turn cause foodcobalamin malabsorption [20].

The FUT2 secretor phenotype was associated with associated with $\mathrm{H}$. pylori infection and with severity of $\mathrm{H}$. pylori-induced gastric lesions [21]. A population-based study in Finland reported high prevalence of $\mathrm{H}$. pylori infection among subjects with chronic atrophic gastritis and low vitamin B12 status [22]. However, a case control study by Stettin et al [23] which examined 90 healthy subjects including 69 with $\mathrm{H}$. pylori positive serology, did not find any significant impact of $\mathrm{H}$. pylori-positive serology on vitamin B status. Similarly, a study of ambulatory subjects from Europe and West Africa found no significant association between $\mathrm{H}$. pylori infection and FUT2 461 G/A (rs601338) polymorphism although FUT2 461 A/A genotype was associated with higher plasma vitamin B12 concentration in the total population [24]. These conflicting studies indicate that the influence of FUT2 polymorphism on vitamin B12 concentration cannot be conclusively linked to $\mathrm{H}$. pylori-related mechanism. Further studies in model systems may elucidate the role of FUT2 polymorphisms on alternative mechanisms like vitamin B12 absorption, transport and cellular uptake that might explain the lower vitamin B12 levels in FUT2 secretor phenotype.

\section{Transcobalamin II}

In circulation, vitamin $\mathrm{B} 12$ is bound to two plasma binding proteins: haptocorrin or transcobalamin . Transcobalamin, the transport protein required for cellular uptake of vitamin B12, constitutes only $20 \%$ of bound plasma vitamin B12. In contrast, haptocorrin binds $\sim 80 \%$ of plasma vitamin B12 but does not facilitate cellular uptake. Specific membrane receptors recognize the protein portion of the transcobalamin-vitamin B12 complex (holo-TC), whereas free vitamin B12 or haptocorrin-bound vitamin B12 (holo$\mathrm{HC}$ ) is not taken up by the cell [25]. Low vitamin B12 concentrations in the cell can be the result of low vitamin B12 intake, but they can also be attributable to a disturbance in the absorption, transport, or cellular uptake of this vitamin. Transcobalamin (TC) is the transporter of vitamin B12 in the circulation and delivers vitamin B12 to the cells. After binding by haptocorrin in the stomach and intrinsic factor in the duodenum, vitamin B12 is transferred to $\mathrm{TC}$ within the enterocyte and released into the blood. Subsequently, the vitamin B12-TC complex is taken up by receptor-mediated endocytosis via the receptor TC-R. Variations in the TC protein could affect the binding characteristics of vitamin B12 to TC or recognition of the vitamin B12-TC complex by transcobalamin receptor (TC-R), with possible repercussions on vitamin B12 availability in the cells. Therefore, genetic variation in the
TC gene may produce altered plasma total homocysteine (tHcy) concentrations and CVD risk [26].

Daiger et al [27] were one of the first to report polymorphic variants of TCN2 in human plasma from Caucasians, American Blacks, and Orientals by PAGE autoradiography. Polymorphic variants were observed in all populations tested; the two most common alleles (of at least four detected) attain frequencies of greater than $40 \%$ in Caucasians and Orientals. They speculated that differences in affinity of the TCN2 allele products or differences in their interaction with cellular receptor sites, may have selective significance and may be involved in maintenance of the TCN2 polymorphism.

The gene encoding TC (TCN2) is located at 22 q11-13.1, spans a region of $18 \mathrm{~kb}$ and has 9 exons [28]. The TCN2 gene shares considerable nucleotide and amino acid sequence homology with the two other cobalamin binding proteins viz. transcobalamin I also known as haptocorrin (TCN1) and with intrinsic factor (IF). Regec et al [29] in a comparative analysis of the placement of introns in the coding regions of human TCN2, TCN1 and IF genes, found substantial homology of the amino acids flanking these insertions and together with previous studies this supports the notion that these genes are derived by duplication of an ancestral gene. The authors suggest that despite this homology, a number of important differences in the TCN2 and TCN1 genes indicated that a major event(s) occurred during this evolutional process that randomly translocated the TCN2 gene to chromosome 22 from chromosome 11, where the human TCN1 and IF genes are located. In addition, the TCN2 gene lacks both the TATA and CCAAT proximal promoter sequences that are contained in the human TCN1 and IF genes, indicating that the translocation of the TCN2 gene did not include the $5^{\prime}$ flanking promoter region. This could account, in part, for the differences in the tissue-specific expression of these proteins.

$\mathrm{Li}$ et al [30] did a sequence analysis of the variant forms of the human TCN2 and suggested that the structural basis for the expression of different polymorphic forms of TCN2 may be due to single point mutations. They described four sequence variants of the human TC gene: M198T, I219L, P259R, and S376L. Variations in the TC protein could affect the binding characteristics of vitamin B12 to $\mathrm{TC}$ or recognition of the vitamin B12-TC complex by TC-R, with possible repercussions on vitamin $\mathrm{B} 12$ availability in the cells. Therefore, genetic variation in the TC gene may produce altered plasma tHcy concentrations and CVD risk [26]. The most common polymorphism in Caucasian populations is a G-to-C substitution at base position $775(775 \mathrm{G}>\mathrm{C})$, which results in the replacement of proline with arginine at codon 259 (P259R). Zetterberg et al (2003) contends that the transcobalamin codon 259 polymorphism should be designated $776 \mathrm{C}>\mathrm{G}$, not $775 \mathrm{G}>\mathrm{C}$ as the polymorphism is a C-to-G substitution at position 776 in relation to the first nucleotide $(+1)$ of the ATG-translation initiation codon, not a G-to-C substitution at nucleotide 775 [31].

Namour et al [32] reported a frequency of $34.6 \%$ for C homozygotes, $47.8 \%$ for heterozygotes, and $17.6 \%$ for $\mathrm{G}$ homozygotes among healthy Caucasians. Heterozygotes 259 P/R were found to have significantly higher plasma homocysteine concentration than homozygotes $259-\mathrm{R}$ and 259-P. They however did not find any significant difference in homocysteine concentration between the homozygotes $\mathrm{P} / \mathrm{P}$ and $\mathrm{R} / \mathrm{R}$. The authors hypothesized that the allele encoding for $\mathrm{P}$ at codon 259 , by enhancing the intracellular vitamin B12 bioavailability, would participate in lowering homocysteine levels, because the active form of vitamin B12, methylcobalamin, is the 
coenzyme of methionine synthase, the enzyme responsible for the methylation of homocysteine to methionine.

Later studies failed to confirm the observation that homocysteine, a functional indicator of B12 status, is higher in heterozygous persons (PR) than in PP and RR persons [33,34].

It has been suggested that the discrepancy between the studies with respect to homocysteine levels is related to differences in the age of the study subjects, with only younger subjects exhibiting the homocysteine difference. A more likely explanation is that the higher homocysteine observed in PR subjects in one study was related to some other uncontrolled determinant of homocysteine, such as sex, B vitamin levels (folate, B12, B6), kidney function, thyroid function, and other genetic factors. In this regard, methylmalonic acid may be better than homocysteine as an indicator of the effect of TCN2 genotype on functional B12 status because methylmalonic acid is influenced by fewer confounding factors [35].

Leivers et al [26] examined five sequence variants, i.e., I23V, G94S, P259R, S348F, and R399Q, in the TC gene as possible determinants of total homocysteine and, concordantly, as possible risk factors for cardiovascular disease in 190 vascular disease patients and 601 controls. They observed amongst individuals with high vitamin B12, 259PP homozygotes had lower tHcy concentrations than 259PR and 259RR heterozygotes. The 259PP genotype, which constitutes a quarter of the general population, may be more susceptible to reduction of plasma tHcy concentrations by increasing the vitamin B12 status. None of the TC genotypes were associated with increased CVD risk. However, in a recent Indian study [36] involving 1398 individuals (589 coronary artery disease (CAD) cases and 809 controls) at least three SNPs in TCN2 gene (G1196A, C776G and $\mathrm{C} 1043 \mathrm{~T}$ ) were associated with CAD in the Indian population and one of these SNPs G1196A with vitamin B12 levels.

Low maternal blood or amniotic fluid cobalamin levels have been associated with NTD risk. In a study involving 46 mothers of children with NTD and 73 control females, all of whom were Dutch Caucasians, Afman et al [33] had shown that low plasma holo TC2 (the proportion of TC2 that contains vitamin B12) concentrations and low holo TC2 percentages are relatively common and increase the risk of NTD three-fold (OR 2.9, 95\% CI 0.9-9.2) and five-fold (OR 5.0, 95\% CI 1.3-19.3) respectively. They however found no significant variation in the frequency of P259R polymorphism between mothers of NTD children (14\%) and controls (21\%). A subsequent study of the same sample population [37] revealed five SNPs by direct sequencing of the coding region of TCN2 gene: I23V, G94S, P259R, S348F and the R399Q. Some of the genotypes defined by these variants influenced total-TC protein content in plasma, holo-TC concentrations and the proportion vitamin $\mathrm{B} 12$ bound to TC: however, no effect on homocysteine and NTD risk was detected. A recent study in India of 318 NTD cases and 702 controls reported maternal $776 \mathrm{C}>\mathrm{G}$ polymorphism in TCN2 to be strongly predictive of NTD in the offspring $(\mathrm{p}=0.006)$ [38].

Vitamin B12 has critical role in normal growth and development, especially so for expectant mothers and older populations. Variant forms of the genes involved in the absorption, transport cellular uptake and metabolism of cobalamin has a bearing on the pathobiology of chronic diseases, particularly involving the intermediate metabolite homocysteine. Information on the genetic defects in these pathways, through large scale association studies in diverse ethnic groups, would help in identifying biomarkers of chronic diseases.

\section{References}

1. Tanaka T, Scheet P, Giusti B, Bandinelli S, Piras MG, et al. (2009) Genome-wide association study of vitamin B6, vitamin B12, folate, and homocysteine blood concentrations. Am J Hum Genet 84: 477-482.

2. VitaminB12-HealthProfessional

3. Marini NJ, Gin J, Ziegle J, Keho KH, Ginzinger D, et al. (2008) The prevalence of folate-remedial MTHFR enzyme variants in humans. Proc Natl Acad Sci U S A 105: 8055-8060.

4. Boushey CJ, Beresford SA, Omenn GS, Motulsky AG (1995) A quantitative assessment of plasma homocysteine as a risk factor for vascular disease. Probable benefits of increasing folic acid intakes. JAMA 274: 1049-1057.

5. Kang SS, Wong PW, Zhou JM, Sora J, Lessick M, et al. (1988) Thermolabile methylenetetrahydrofolate reductase in patients with coronary artery disease. Metabolism 37: 611-613.

6. Kluijtmans LA, van den Heuvel LP, Boers GH, Frosst P, Stevens EM, et al. (1996) Molecular genetic analysis in mild hyperhomocysteinemia: a common mutation in the methylenetetrahydrofolate reductase gene is a genetic risk factor for cardiovascular disease. Am J Hum Genet 58: 35-41.

7. Chambers JC, Obeid OA, Refsum H, Ueland P, Hackett D, et al. (2000) Plasma homocysteine concentrations and risk of coronary heart disease in UK Indian Asian and European men. Lancet 355: 523-527.

8. Schneider JA, Rees DC, Liu YT, Clegg JB (1998) Worldwide distribution of a common methylenetetrahydrofolate reductase mutation. Am J Hum Genet 62: 1258-1260.

9. Chen C, Gan YY (2010) The allele frequencies of three polymorphisms in genes involved in homocysteine metabolism in a group of unrelated healthy Singaporeans. Dis Markers 29: 111-119.

10. Yakub M, Moti N, Parveen S, Chaudhry B, Azam I, et al. (2012) Polymorphisms in MTHFR, MS and CBS genes and homocysteine levels in a Pakistani population. PLoS One 7: e33222.

11. Yan L, Zhao L, Long Y, Zou P, Ji G, et al. (2012) Association of the maternal MTHFR C677T polymorphism with susceptibility to neural tube defects in offsprings: evidence from 25 case-control studies. PLoS One 7: e41689.

12. van der Put NM, Gabreëls F, Stevens EM, Smeitink JA, Trijbels FJ, et al. (1998) A second common mutation in the methylenetetrahydrofolate reductase gene: an additional risk factor for neural-tube defects? Am J Hum Genet 62: 1044-1051.

13. Wang XW, Luo YL, Wang W, Zhang Y, Chen Q, et al. (2012) Association between MTHFR A1298C polymorphism and neural tube defect susceptibility: a metaanalysis. Am J Obstet Gynecol 206: 251.

14. Larsen RD, Ernst LK, Nair RP, Lowe JB (1990) Molecular cloning, sequence, and expression of a human GDP-L-fucose:beta-D-galactoside 2-alpha-L-fucosyltransferase cDNA that can form the $\mathrm{H}$ blood group antigen. Proc Natl Acad Sci U S A 87: 6674-6678.

15. Kelly RJ, Rouquiers S, Giorgi D, Lennons GG, Lowe JB (1995) Sequence and Expression of a Candidate for the Human Secretor Blood Group I $\pm(1,2)$ Fucosyltransferase Gene (FUT2). J Biol Chem 270: 4640-4649.

16. Koda Y, Tachida H, Pang H, Liu Y, Soejima M, et al. (2001) Contrasting patterns of polymorphisms at the ABO-secretor gene (FUT2) and plasma alpha(1,3)fucosyltransferase gene (FUT6) in human populations. Genetics 158: 747-756.

17. Hazra A, Kraft P, Lazarus R, Chen C, Chanock SJ, et al. (2009) Genomewide significant predictors of metabolites in the one-carbon metabolism pathway. Hum Mol Genet 18: 4677-4687.

18. Hazra A, Kraft P, Selhub J, Giovannucci EL, Thomas G, et al. (2008) Common variants of FUT2 are associated with plasma vitamin B12 levels. Nat Genet 40: 1160-1162. 
Citation: Das D, Haloi A (2014) Vitamin B12 Gene Polymorphisms and Chronic Diseases. J Nutr Disorders Ther 4: 149. doi: 10.4172/2161-0509-4.1000149

Page 5 of 5

19. Lin X, Lu D, Gao Y, Tao S, Yang X, et al. (2012) Genome-wide association study identifies novel loci associated with serum level of vitamin B12 in Chinese men. Hum Mol Genet 21: 2610-2617.

20. Carmel R, Aurangzeb I, Qian D (2001) Associations of food-cobalamin malabsorption with ethnic origin, age, Helicobacter pylori infection, and serum markers of gastritis. Am J Gastroenterol 96: 63-70.

21. Azevedo M, Eriksson S, Mendes N, Serpa J, Figueiredo C, et al. (2008) Infection by Helicobacter pylori expressing the BabA adhesin is influenced by the secretor phenotype. J Pathol 215: 308-316.

22. Sipponen P, Laxén F, Huotari K, Härkönen M (2003) Prevalence of low vitamin B12 and high homocysteine in serum in an elderly male population: association with atrophic gastritis and Helicobacter pylori infection. Scand J Gastroenterol 38: 1209-1216.

23. Stettin D, Waldmann A, Ströhle A, Hahn A (2008) Association between Helicobacter pylori-infection, C-reactive protein and status of $\mathrm{B}$ vitamins. Adv Med Sci 53: 205-213.

24. Oussalah A, Besseau C, Chery C, Jeannesson E, Rosa-Maria GueantRodriguez R-M et al. (2012) Helicobacter pylori serologic status has no influence on the association between fucosyltransferase 2 polymorphism (FUT2 461 G/A) and vitamin B-12 in Europe and West Africa. Am J Clin Nutr 95: 514-521.

25. von Castel-Dunwoody KM1, Kauwell GP, Shelnutt KP, Vaughn JD, Griffin ER, et al. (2005) Transcobalamin 776C->G polymorphism negatively affects vitamin B-12 metabolism. Am J Clin Nutr 81: 1436-1441.

26. Lievers KJ, Afman LA, Kluijtmans LA, Boers GH, Verhoef P, et al. (2002) Polymorphisms in the transcobalamin gene: association with plasma homocysteine in healthy individuals and vascular disease patients. Clin Chem 48: 1383-1389.

27. Daiger SP, Labowe ML, Parsons M, Wang L, Cavalli-Sforza LL (1978) Detection of genetic variation with radioactive ligands. III. genetic polymorphism of transcobalamin II in human plasma. Am J Hum Genet 30: 202-214.

28. Quadros EV (2010) Advances in the understanding of cobalamin assimilation and metabolism. Br J Haematol 148: 195-204.
29. Regec A, Quadros EV, Platica O, Rothenberg SP (1995) The cloning and characterization of the human transcobalamin II gene. Blood 85: 2711-2719.

30. Li N, Seetharam S, Lindemans J, Alpers DH, Arwert F, et al. (1993) Isolation and sequence analysis of variant forms of human transcobalamin II. Biochim Biophys Acta 1172: 21-30.

31. Zetterberg H, Palmer M, Boreström C, Rymo L, Blennow K (2003) The transcobalamin codon 259 polymorphism should be designated $776 \mathrm{C}>\mathrm{G}$, not 775G>C. Blood 101: 3749-3750.

32. Namour F, Olivier J, Abdelmouttaleb I, Adjalla C, Debard R, et al. (2001) Transcobalamin codon 259 polymorphism in HT-29 and Caco-2 cells and in Caucasians: relation to transcobalamin and homocysteine concentration in blood. Blood 97: 1092-1098.

33. Afman LA, Van Der Put NM, Thomas CM, Trijbels JM, Blom HJ (2001) Reduced vitamin B12 binding by transcobalamin II increases the risk of neural tube defects. QJM 94: 159-166.

34. McCaddon A, Blennow K, Hudson P, Regland B, Hill D (2001) Transcobalamin polymorphism and homocysteine. Blood 98: 3497-3499.

35. Miller JW, Ramos MI, Garrod MG, Flynn MA, Green R (2002) Transcobalamin II 775G $>$ C polymorphism and indices of vitamin B12 status in healthy older adults. Blood 100: 718-720.

36. Garg G, Kumar J, Tanwar VS, Basak T, Seth S, et al. (2012) Polymorphisms in transcobalamin II gene is associated with coronary artery disease in Indian population. Biomarkers 17: 119-124.

37. Afman LA, Lievers KJ, van der Put NM, Trijbels FJ, Blom HJ (2002) Single nucleotide polymorphisms in the transcobalamin gene: relationship with transcobalamin concentrations and risk for neural tube defects. Eur J Hum Genet 10: 433-438.

38. Godbole K, Gayathri P, Ghule S, Sasirekha BV, Kanitkar-Damle A, et al. (2011) Maternal one-carbon metabolism, MTHFR and TCN2 genotypes and neural tube defects in India. Birth Defects Res A Clin Mol Teratol 91: 848-856. 